\title{
FIRST SPECTROSCOPIC IMAGING OBSERVATIONS OF THE SUN AT LOW RADIO FREQUENCIES WITH THE MURCHISON WIDEFIELD ARRAY PROTOTYPE
}

\author{
Divya Oberoi ${ }^{1}$, Lynn D. Matthews ${ }^{1}$, Iver H. Cairns ${ }^{2}$, David Emrich ${ }^{3}$, Vasili Lobzin ${ }^{2}$, Colin J. Lonsdale ${ }^{1}$, \\ Edward H. Morgan ${ }^{4}$, T. Prabu ${ }^{5}$, Harish Vedantham ${ }^{5}$, Randall B. Wayth ${ }^{3}$, Andrew Williams ${ }^{6}$, Christopher Willliams ${ }^{4}$, \\ Stephen M. White ${ }^{7}$, G. Allen ${ }^{8}$, Wayne Arcus ${ }^{3}$, David Barnes ${ }^{9}$, Leonid Benkevitch ${ }^{1}$, Gianni Bernardi ${ }^{10}$, \\ Judd D. Bowman ${ }^{11}$, Frank H. Briggs ${ }^{12}$, John D. Bunton ${ }^{8}$, Steve Burns ${ }^{13}$, Roger C. Cappallo ${ }^{1}$, M. A. Clark ${ }^{14}$, \\ Brian E. Corey ${ }^{1}$, M. Dawson ${ }^{12}$, David DeBoer ${ }^{8,15}$, A. De Gans ${ }^{12}$, Ludi DeSouza ${ }^{8}$, Mark Derome ${ }^{1}$, R. G. Edgar ${ }^{14,16}$, \\ T. Elton ${ }^{8}$, Robert Goeke $^{4}$, M. R. Gopalakrishna ${ }^{5}$, Lincoln J. Greenhill ${ }^{10}$, Bryna Hazelton ${ }^{17}$, David Herne ${ }^{3}$, \\ JaCQueline N. HewitT $^{4}$, P. A. Kamini ${ }^{5}$, David L. Kaplan ${ }^{18}$, Justin C. KasPer ${ }^{10}$, Rachel Kennedy ${ }^{1,15}$, Barton B. KinCaid ${ }^{1}$, \\ Jonathan Kocz ${ }^{12}$, R. Koeing ${ }^{8}$, Errol Kowald ${ }^{12}$, Mervyn J. Lynch ${ }^{3}$, S. Madhavi ${ }^{5}$, Stephen R. McWhirter ${ }^{1}$, \\ Daniel A. Mitchell ${ }^{10}$, Miguel F. Morales ${ }^{17}$, A. NG ${ }^{8}$, Stephen M. Ord $^{10}$, Joseph Pathikulangara ${ }^{8}$, Alan E. E. Rogers ${ }^{1}$, \\ Anish Roshi ${ }^{5}, 19$, Joseph E. Salah ${ }^{1}$, Robert J. Sault ${ }^{20}$, Antony Schinckel ${ }^{8}$, N. Udaya Shankar ${ }^{5}$, K. S. SRivani ${ }^{5}$, \\ Jamie Stevens ${ }^{8}$, Ravi Subrahmanyan ${ }^{5}$, D. Thakkar ${ }^{2}$, Steven J. TingaY ${ }^{3}$, J. Tuthill ${ }^{8}$, Annino Vaccarella ${ }^{12}$, \\ MARK WATERSON $^{3,12}$, RAChel L. WebsteR ${ }^{20}$, AND ALAN R. WhitNeY ${ }^{1}$ \\ ${ }^{1}$ MIT Haystack Observatory, Westford, MA, USA \\ ${ }^{2}$ School of Physics, University of Sydney, Sydney, Australia \\ ${ }^{3}$ Curtin Institute for Radio Astronomy, Curtin University, Perth, Australia \\ ${ }^{4}$ MIT Kavli Institute for Astrophysics and Space Research, Cambridge, MA, USA \\ ${ }^{5}$ Raman Research Institute, Bangalore, India \\ ${ }^{6}$ Perth Observatory, The University of Western Australia, Perth, Australia \\ ${ }^{7}$ Air Force Research Laboratory, Kirtland, NM, USA \\ ${ }^{8}$ CSIRO Astronomy and Space Science, Epping, NSW, Australia \\ ${ }^{9}$ Center for Astrophysics and Supercomputing, Swinburne University of Technology, Melbourne, Australia \\ ${ }^{10}$ Harvard-Smithsonian Center for Astrophysics, Cambridge, MA, USA \\ ${ }^{11}$ School of Earth and Space Exploration, Arizona State University, Tempe, AZ, USA \\ ${ }^{12}$ Research School of Astronomy and Astrophysics, The Australian National University, Canberra, Australia \\ ${ }_{13}$ Burns Industries, Inc., Nashua, NH, USA \\ ${ }^{14}$ Faculty of Arts and Sciences, Harvard University, Cambridge, MA, USA \\ ${ }^{15}$ Berkeley Astronomy Department, University of California, Berkeley, CA, USA \\ ${ }^{16}$ Massachusetts General Hospital, Boston, MA, USA \\ ${ }^{17}$ Department of Physics, University of Washington, Seattle, WA, USA \\ ${ }^{18}$ Department of Physics, University of Wisconsin-Milwaukee, Milwaukee, WI, USA \\ ${ }^{19}$ National Radio Astronomy Observatory, Green Bank, WV, USA \\ ${ }^{20}$ School of Physics, The University of Melbourne, Melbourne, Australia \\ Received 2010 December 3; accepted 2010 December 28; published 2011 January 25
}

\begin{abstract}
We present the first spectroscopic images of solar radio transients from the prototype for the Murchison Widefield Array, observed on 2010 March 27. Our observations span the instantaneous frequency band 170.9201.6 MHz. Though our observing period is characterized as a period of "low" to "medium" activity, one broadband emission feature and numerous short-lived, narrowband, non-thermal emission features are evident. Our data represent a significant advance in low radio frequency solar imaging, enabling us to follow the spatial, spectral, and temporal evolution of events simultaneously and in unprecedented detail. The rich variety of features seen here reaffirms the coronal diagnostic capability of low radio frequency emission and provides an early glimpse of the nature of radio observations that will become available as the next generation of low-frequency radio interferometers come online over the next few years.
\end{abstract}

Key words: instrumentation: interferometers - radiation mechanisms: non-thermal - Sun: corona - Sun: radio radiation

Online-only material: animations

\section{INTRODUCTION}

Low radio frequency $(v \lesssim 300 \mathrm{MHz})$ emission provides powerful diagnostics of the solar corona. However, high-fidelity solar imaging at low frequencies is challenging. Coronal emission features are complex and dynamic, evolving rapidly in space, time, and frequency. Consequently, the limited instantaneous spatial and frequency coverage provided by current radio interferometers have been inadequate to simultaneously resolve transient solar phenomena spatially, temporally, and spectrally.
This situation should change dramatically in the next few years as a new generation of low-frequency radio arrays becomes available, leveraging recent advances in digital signal processing hardware and computational capacity. The Murchison Widefield Array (MWA; Lonsdale et al. 2009) will be one such array, with most of its 512 elements spread over $1.5 \mathrm{~km}$ and a few outliers out to $3 \mathrm{~km}$. The resulting dense instantaneous monochromatic $u v$ coverage will provide a radio imaging capability with unprecedented fidelity and flexibility. The MWA is currently under construction at the Murchison Radioastronomy 


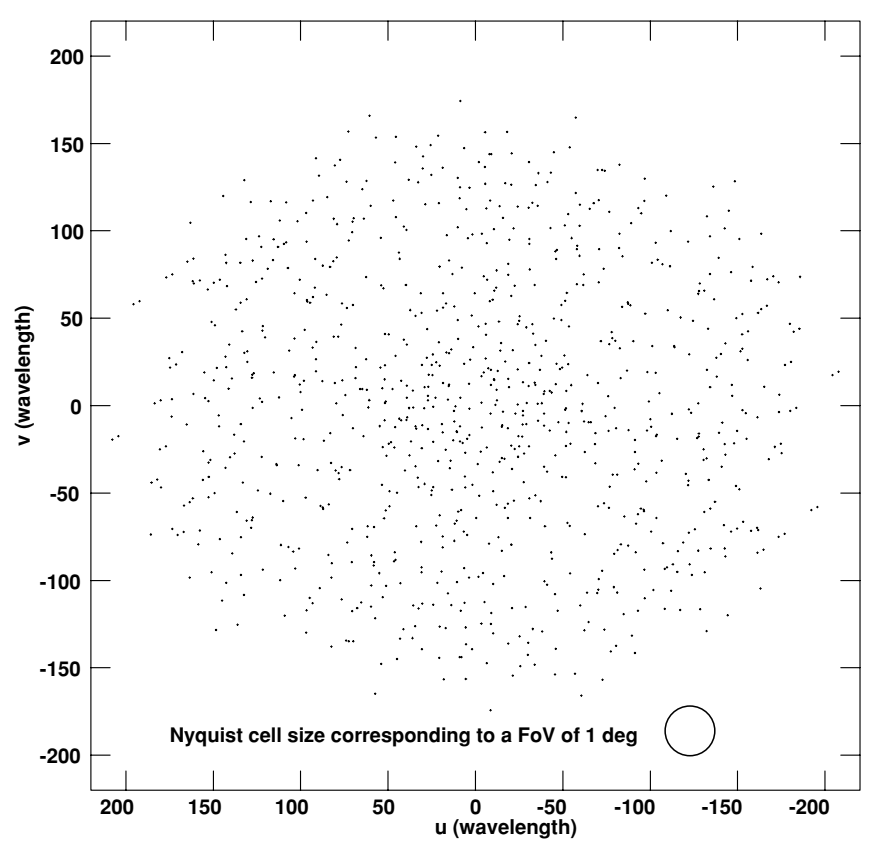

Figure 1. Instantaneous $u v$ coverage for a single coarse channel centered at 186.2 MHz. The circle indicates the size of a $u v$-cell, corresponding to the Nyquist sampling criterion for a $1^{\circ} \mathrm{FoV}$.

Observatory, in the remote and radio-quiet Western Australian outback, and a prototype system comprising 32 interferometer elements (tiles) is operational on site. This system, hereafter referred to as the "32T," serves as an engineering test bed and provides early science opportunities, in advance of the full MWA. We present here solar imaging observations obtained with the 32T. The quiescent solar emission in the MWA frequency range of $80-300 \mathrm{MHz}$ is dominated by coronal emission from heights of $\sim 1-10 R_{\odot}$ above the photosphere. These are among the first high-fidelity, high-dynamic-range, spectroscopic images of the Sun with a good temporal and spectral resolution at meter wavelengths.

\section{DATA}

The 32T tiles are arranged along the arms of a randomized Reuleaux triangle (see Cohanim et al. 2004), providing a fairly uniformly sampled $u v$ plane with baseline lengths up to $350 \mathrm{~m}$ (Figure 1). For solar observations, the correlated flux is dominated by the Sun, reducing the effective field of view (FoV) to $\sim 1^{\circ}$, for which the $u v$ sampling provided by the $32 \mathrm{~T}$ exceeds the Nyquist criterion. ${ }^{21}$ This, together with a high signal-tonoise ratio, permits robust, high-fidelity imaging (Bracewell \& Roberts 1954).

In its usual interferometric mode of operation, the $32 \mathrm{~T}$ provides auto- and cross-correlations (visibilities) for all 64 input signals ( 32 tiles $\times 2$ linear polarizations). The entire radio frequency band is directly sampled and filtered into $1.28 \mathrm{MHz}$ wide coarse channels. Twenty-four of these coarse channels are further processed by the correlator, which can currently provide a time resolution of $50 \mathrm{~ms}$ with a $50 \%$ duty cycle over a $30.72 \mathrm{MHz}$ band and a spectral resolution of $40 \mathrm{kHz}$. The 32T design closely follows the MWA architecture described in Lonsdale et al. (2009).

${ }^{21} \delta u \sim 1 / 2 \theta_{0}$, where $\delta u$ is the length scale in the $u v$ plane beyond which visibilities are no longer correlated and $\theta_{0}$ is the size of the FoV in radians.
The data presented here were obtained on 2010 March 27 from 04:24:53 to 04:34:03 UT and span the range 170.8-201.6 MHz. They were smoothed to a time resolution of $1 \mathrm{~s}$ before further processing. Data editing, calibration, and imaging were performed using the Astronomical Image Processing System. Self-calibration, to solve for frequency-independent complex gains, was performed individually for each of the coarse channels using a $10 \mathrm{~s}$ interval where the Sun was in a relatively quiescent state. These gain solutions were then applied to the full time interval. No absolute flux calibration was possible, owing to the lack of observations of a suitable calibrator. The amplitude part of the bandpass was calibrated using the total power spectra from the same quiescent time interval as used for self-calibration. As will become evident later, the choice of a spectral index only impacts the underlying spectral slope. We assumed a spectral index $\alpha=2.6$ for the quiet Sun $\left(S_{v} \propto v^{\alpha}\right.$, where $S_{v}$ is the flux density and $v$ is the observing frequency); this choice is based on $32 \mathrm{~T}$ observations during the recent deep solar minimum (2008 November), which yielded $\alpha=2.6 \pm 0.4$, a value consistent with Erickson et al. (1977). All the data presented here correspond to the east-west (XX) polarization. The wide FoV MWA tiles are expected to have significant (but stable) cross-polarization leakage, and an absolute polarization calibration was not attempted.

Imaging and deconvolution were performed using the standard CLEAN algorithm with robust $(\mathcal{R}=0)$ weighting, resulting in a synthesized beam at the band center of $961^{\prime \prime} \times 796^{\prime \prime}$ (FWHM). For the images presented here, the data were averaged over a single coarse channel during the gridding process and restored with a circular beam with FWHM 800". The edges of the coarse channels could not be calibrated satisfactorily, hence the first six and the last four spectral channels of each coarse channel were flagged during imaging.

\section{ANALYSIS AND RESULTS}

Amplitudes and phases observed on a representative short ( $\sim 64 \mathrm{~m})$ and long $(\sim 315 \mathrm{~m})$ projected baseline are shown in Figure 2. Baselines of different lengths and orientations measure independent Fourier components of the source structure. The examples shown here illustrate several key features of the data. Only one polarization is shown as both polarizations show very similar behavior. The most prominent feature in Figure 2, visually obvious on all baselines in both amplitude and phase, spans the entire observing band around 04:30:10 UT. We hereafter refer to this as the "broadband" feature. Its properties are similar to a weak type III burst (Section 4). In addition, a large number of shorter-lived, narrowband features are evident in the visibility amplitudes. Many of these amplitude variations are accompanied by corresponding variations in phase, implying a change in the brightness temperature $\left(T_{B}\right)$ distribution in the corona. These features become more numerous and prominent visually with increasing baseline length and are referred to as "narrowband" features hereafter. A dearth of truly "quiescent" periods is also evident, and the ubiquitous modest variations seen in the spectral structure are solar, not instrumental, in origin.

To further illustrate the diversity in the types of emission seen in the short span of data presented here, as well as its spectrally complex and highly time-variable behavior, Figure 3 shows several series of autocorrelation (total power) spectra. The bottom panel shows one of the few quiescent intervals; the baseline spectral slope seen here is due to the spectral index of the quiet Sun, dominated by thermal emission. An amplitude 

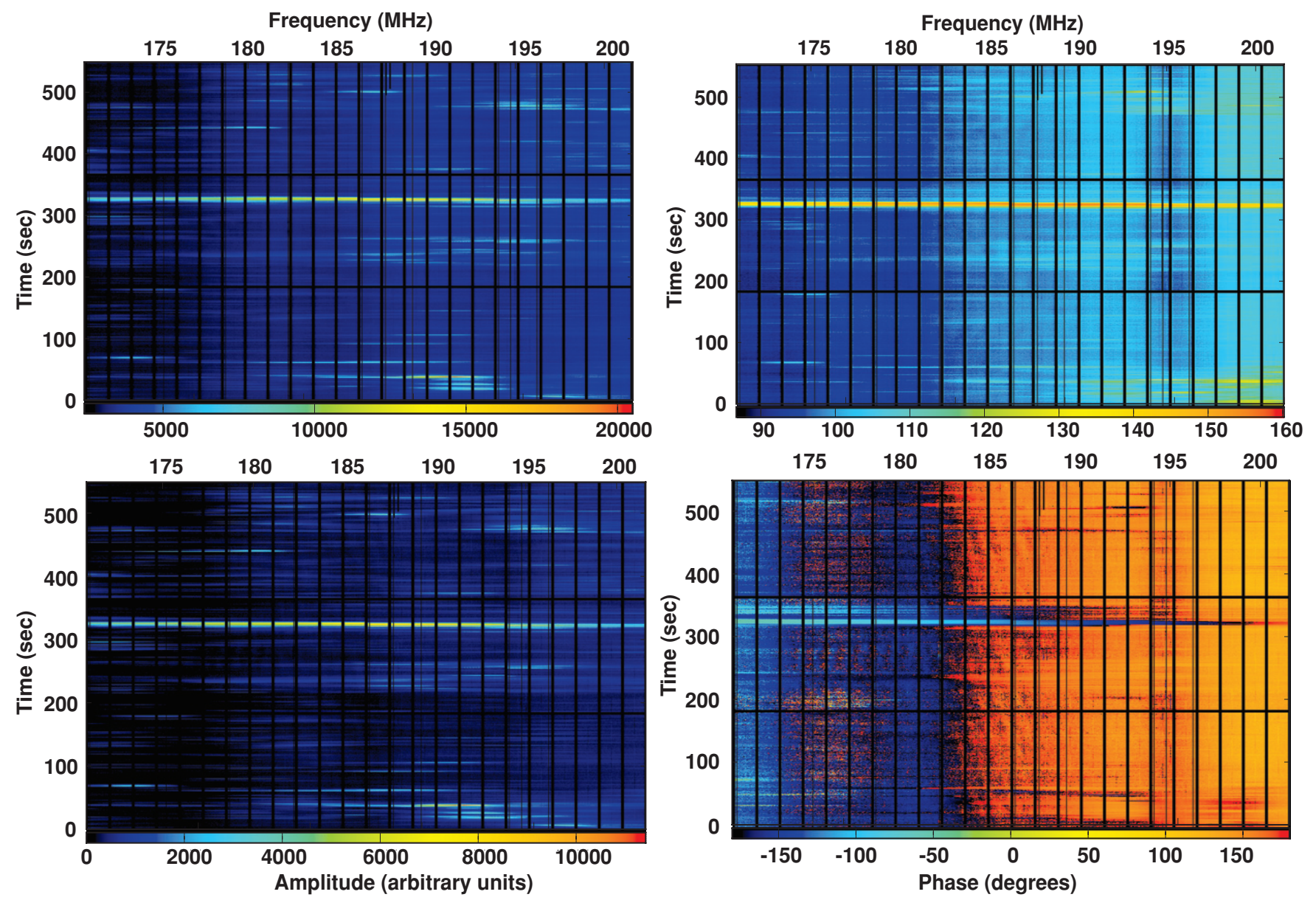

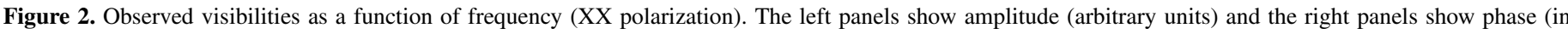

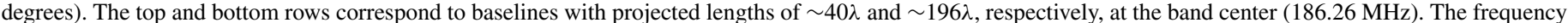

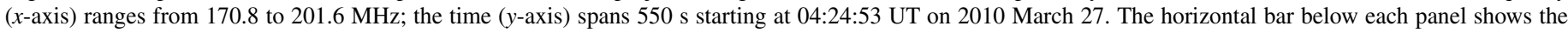
color-coded scale and the dark vertical streaks arise from flagging of the coarse channel edges (Section 2).

variation of $\sim 5 \%$ over a 10 s interval is seen even in "featureless" parts of the data. The middle panel shows a series of spectra illustrating one of the numerous "narrowband" features seen in Figure 2. These features show remarkably rapid evolution in spectral shape and intensity. They typically span $\sim 5-10 \mathrm{MHz}$ in bandwidth, outside of which the spectral flux density returns to that of the quiet Sun. Their peak flux density approaches $\sim 1.5$ times the quiescent solar flux density at that frequency. Finally, the top panel of Figure 3 shows the dynamic behavior of the "broadband" feature around 04:30:10 UT. Its bandwidth exceeds the $\sim 30 \mathrm{MHz}$ observing bandwidth, and at its peak, its flux density is $\sim 2.5$ times the quiescent solar flux density. The abrupt changes from one second to the next imply that the physical changes leading to the production of this emission are temporally undersampled.

The data presented here permit a high-fidelity image of the Sun for every individual time and frequency slice (i.e., every pixel on the dynamic spectra shown in Figure 2). Figure 4 shows a collage of images that illustrates the rapid evolution of the appearance of the solar corona during our observations. Although the spatial resolution of the $32 \mathrm{~T}$ is limited, the fidelity and dynamic range of these images is unprecedented at these frequencies; the dynamic range of $\sim 2500$ exceeds that of earlier images by about an order of magnitude (cf. Mercier et al. 2006; Mercier \& Chambe 2009). The higher fidelity and dynamic range enables us not only to image the quiescent solar emission in the presence of much brighter features, unlike most earlier observations (e.g., Kai 1970; Kundu et al. 1986; Krucker et al. 1995; Vilmer et al. 2002; Ramesh et al. 2010), but also to track low-level variations in the coronal emission. The bottom panels in Figure 4 illustrate the morphological relationship between our $32 \mathrm{~T}$ radio images and the extreme ultraviolet (EUV) images taken on the same day. A persistent feature coinciding with the location of the NOAA Space Weather Prediction Center (SWPC) region 11057 (hereafter region 11057) is seen in the solar radio images, even at quiescent times.

Rapid and significant changes in the radio morphology of the Sun on timescales of seconds are clearly visible in the images presented in Figure 4 and are further highlighted in the two "movies" available in the online journal. A significant fraction of this variability maps back to the location of the region 11057. Subtle changes in $T_{B}$ as a function of time are also discernible at the locations of the active regions on the eastern limb and close to the center of the disk.

Figure 5 highlights the spectroscopic capability of the 32T by showing the spectra from representative pixels across the entire emitting region. Various interesting features can be seen here. Even at quiescent times, there is a significant difference in the spectral slope of regions close to and away from region 11057. For both the "narrowband" and "broadband" features, the changes in spectral structure are confined to the vicinity of region 11057 , and to a smaller extent to the locations of other 


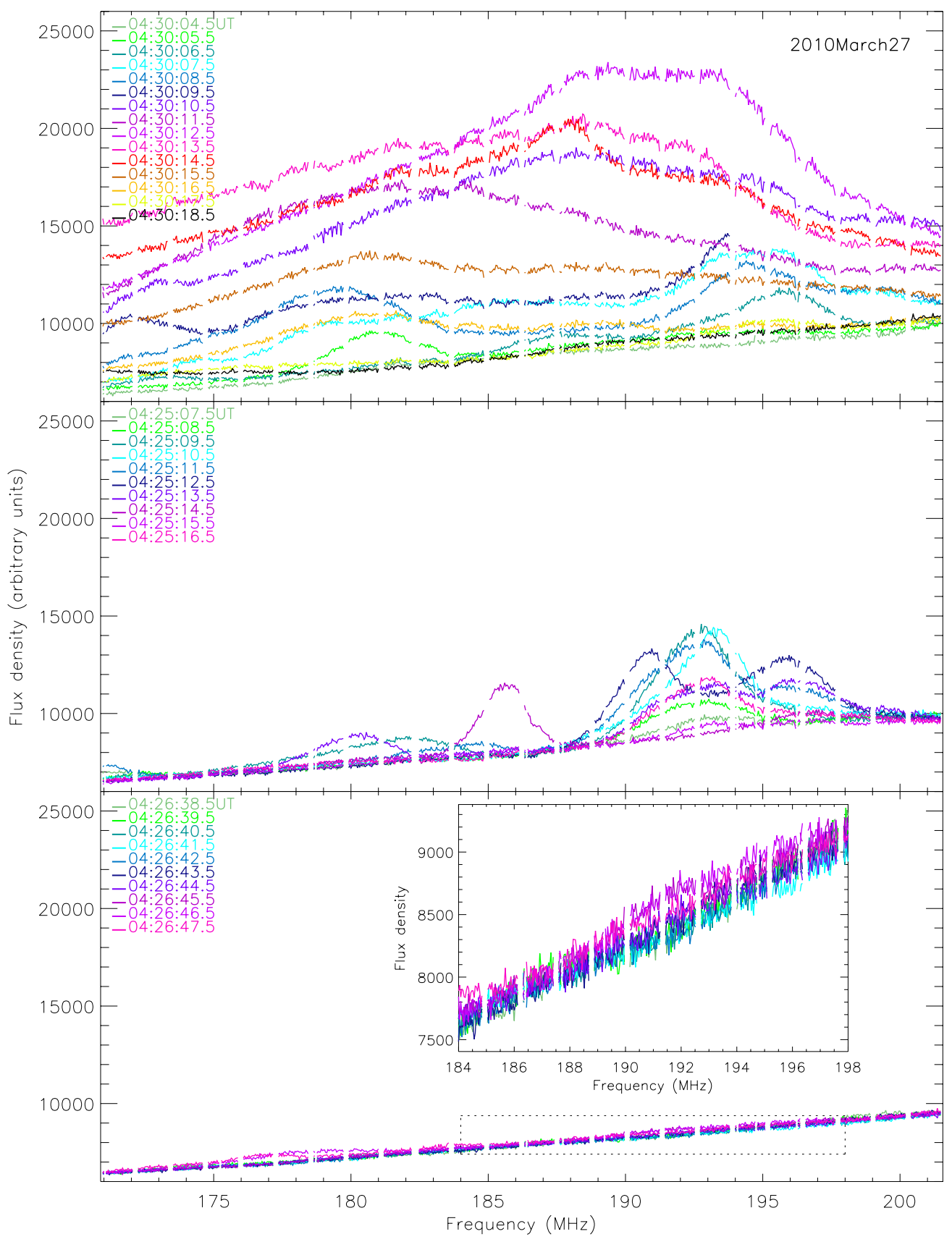

Figure 3. Sample autocorrelation spectra from tile 6, X polarization. Top panel: a 14 s period bracketing the most prominent "broadband" feature seen in Figure 2. Middle panel: variations seen across $10 \mathrm{~s}$ for one of the numerous shorter-lived "narrowband" intensity enhancements. Bottom panel: a $10 \mathrm{~s}$ interval exhibiting little intensity variation. The inset in the bottom panel zooms in on a part of the band. As with Figure 2, the data gaps reflect flagged data.

active regions visible in the EUV image (Figure 4). The "narrowband" features are consistent with spectral features superposed on the underlying quiescent spectrum. In contrast, the underlying quiescent spectrum is no longer visible in spectra of the "broadband" feature, and the changes in spectral characteristics are significantly larger, more dynamic, and complex.

\section{DISCUSSION AND CONCLUSIONS}

At low radio frequencies, a featureless continuum due to thermal bremsstrahlung from a corona with optical depth approaching unity constitutes the bulk of the "quiet" Sun emission and is expected to vary only slowly in time (e.g., McLean \& Sheridan 1985). In this regime, emission that exhibits rapid spectral variations is dominated by plasma emission, a resonant emission mechanism in which electrostatic Langmuir waves are converted to electromagnetic radiation at the local plasma frequency, $f_{P}$, and its harmonic, $2 f_{P}$ (e.g., Robinson \& Cairns 2000). This is the accepted emission mechanism for type II (e.g., Nelson \& Melrose 1985) and type III solar bursts (e.g., Suzuki \& Dulk 1985).

The ability of the $32 \mathrm{~T}$ to image and track the temporal and spectral evolution of coronal emission with high dynamic range and fidelity over a broad spectral band represents a major advance in the quality, and consequently the utility, of radio measurements for understanding the dynamic solar corona. For example, we see numerous instances of transient, nonthermal emission in our short observations, many of which outshine the Sun by a factor of few and dramatically alter 


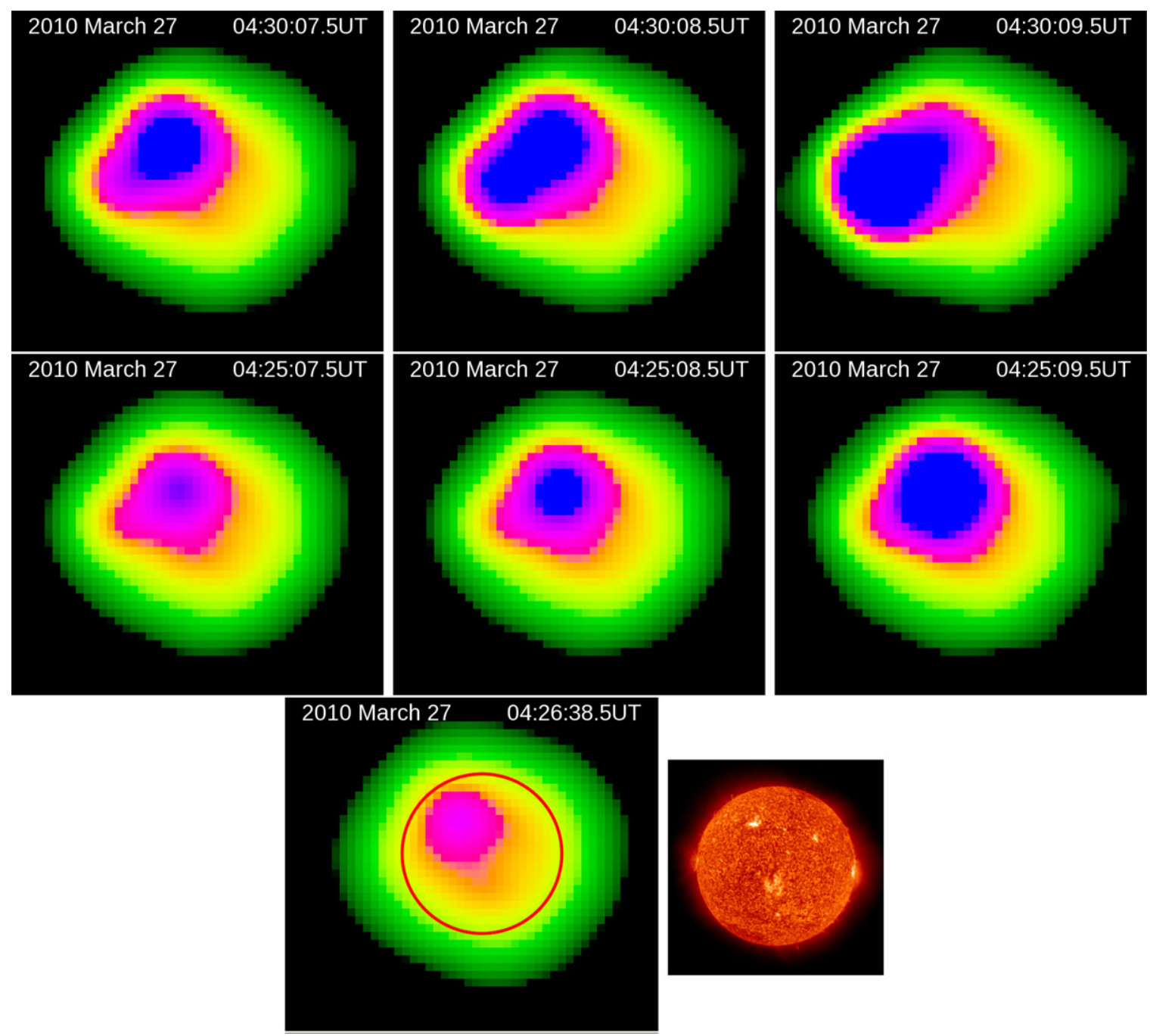

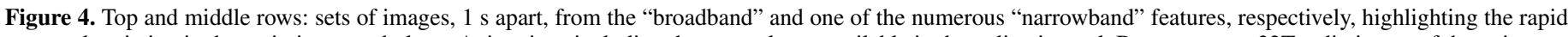

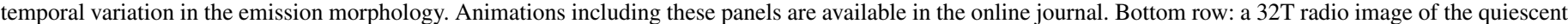

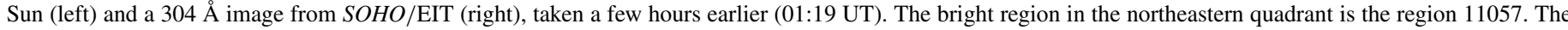

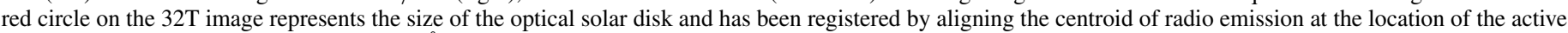

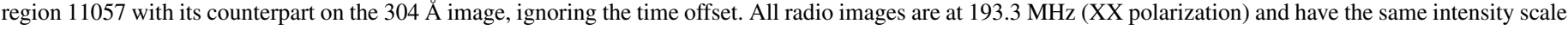
(0-2500, arbitrary units). All images have celestial north on top.

(Animations $[\mathrm{A}, \mathrm{B}]$ of this figure are available in the online journal.)

the $T_{B}$ distribution. Though there have been earlier imaging observations of solar radio transients, these investigations have been limited to a handful of well-separated, non-contiguous frequencies due to the limited spectral sampling provided by previous radio interferometers.

The coherent emission mechanisms inherent at low radio frequencies have long been known to be a sensitive probe of electron acceleration in the corona. Given our much improved observing capability, it is not a surprise that in spite of significant transient behavior seen in our data, the level of solar activity on the day of our observations has been characterized as $l o w^{22}$ to medium. ${ }^{23}$ The SWPC "events list" reports nine type III radio bursts on this day, none of them during the interval presented here. The SWPC also reports seven GOES B-class and four GOES C-class X-flares on this day (ten and five X-ray flares, respectively, in a $24 \mathrm{hr}$ period around our observations). All of the flares for which positional information is available

\footnotetext{
22 USAF/NOAA Solar Geophysical "Weekly" Data.

23 http://www.solarmonitor.org
}

(C-class) were associated with the region 11057 . The start time for one of the B-class flares coincides with the stop time of our observations.

Based on its appearance in the frequency-time plane, the "broadband" feature present in our data (Figure 2) resembles a weak type III burst and shares similarities with the "microbursts" reported by Kundu et al. (1986). A corresponding feature was seen at low signal-to-noise levels between $\sim 80$ and $150 \mathrm{MHz}$ by the Learmonth and Culgoora radiospectrographs, suggesting that this feature peaked in intensity within that spectral window. Though it appears to be a feature with a fast spectral drift rate, the signal-to-noise ratio and temporal sampling of the Learmonth and Culgoora data are not sufficient for a reliable estimate of the drift rate. Nançay Radioheliograph data spanning the wide 150-455 MHz frequency band show two persistent but time-variable features separated by $\sim 200^{\prime \prime}$ associated with region 11057 at nearby times. This suggests the presence of an underlying weak type IV emission. The non-thermal spectra of the region 11057 even at "quiescent" times and the persistent feature seen at this location in our data are consistent with this. 


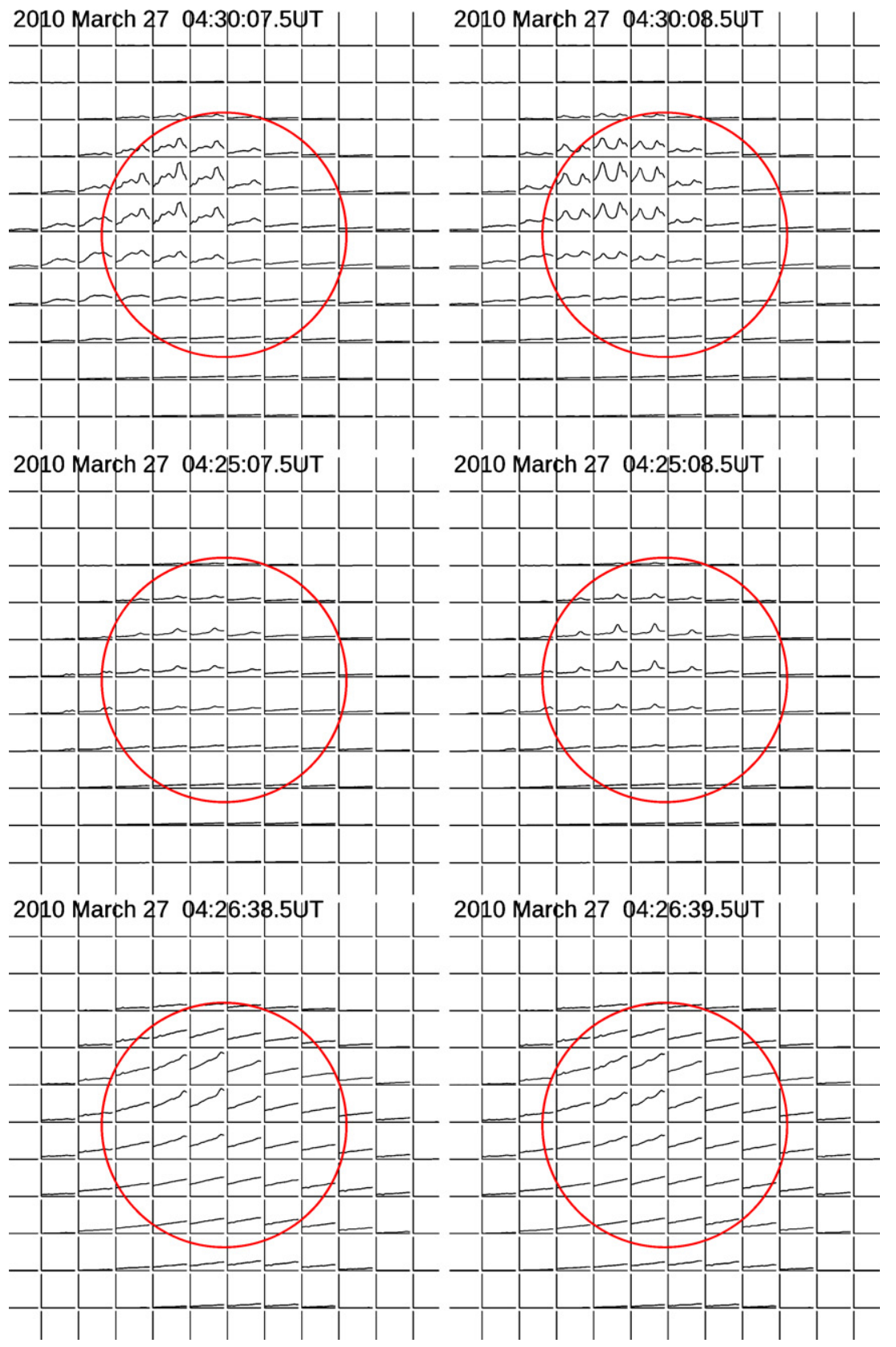

Figure 5. Spatially localized spectra of emission across the solar disk at several of the same times shown in Figure 4. Celestial north is on top, and the red circle marks the size of the optical solar disk. Spectra are shown for every third pixel, where the pixel size is $100^{\prime \prime} \times 100^{\prime \prime}$. The spectra span the entire observing bandwidth, binned into 24 frequency bands spaced $1.28 \mathrm{MHz}$ apart and averaged over $0.8 \mathrm{MHz}$. The $y$-axis ranges are (in arbitrary units) - top row: 50-4996 and 50-4620, respectively; middle row: 100-6000 for both panels; bottom row: 100-1800 for both panels. Animations showing additional frames are available in the online journal. The frames corresponding to the bottom two rows have fixed $y$-axis scales; those for the top row are autoscaled.

(Animations $[\mathrm{A}, \mathrm{B}, \mathrm{C}]$ of this figure are available in the online journal.)

Lacking an absolute flux calibration, we compute an approximate $T_{B}$ of the wideband burst at the center of our observing band by assigning the "quiet" Sun a $T_{B}$ of $\approx 7.5 \times 10^{5} \pm 15 \% \mathrm{~K}$, based on measurements of Lantos \& Avignon (1975) at $169 \mathrm{MHz}$, and scaling it to the peak of the burst emission (04:30:12.5 UT). A measured receiver temperature of $\sim 50 \mathrm{~K}$ and an estimated Galactic background contribution of $\sim 150 \mathrm{~K}$ were used. We estimate an average $T_{B} \sim 3.1 \times 10^{6} \mathrm{~K}$, significantly lower than the average value of $6.3 \times 10^{7} \mathrm{~K}$ reported for type III bursts at $169 \mathrm{MHz}$ (Suzuki \& Dulk 1985). The low value of $T_{B}$ measured highlights the ability of the $32 \mathrm{~T}$ to make sensitive measurements. We note that since the angular size of the emitting region is expected to be much smaller than the $32 \mathrm{~T}$ beam, the measured $T_{B}$ is expected to be considerably reduced due to beam dilution. Assuming a plasma emission process and the Newkirk $(10 \times$ Newkirk) model (Newkirk 1961) for coronal electron density leads to a coronal height of $1.7 R_{\odot}$ $\left(2.8 R_{\odot}\right)$ above the photosphere for the source region at our central observing frequency. A bandwidth of $30 \mathrm{MHz}$ corresponds to a range of $\sim 0.09 R_{\odot}\left(\sim 0.25 R_{\odot}\right)$ around this coronal height.

A particularly intriguing aspect of our data is the abundance of short-lived, "narrowband" emission features carpeting the frequency-time plane, which to our knowledge, have not been 
reported previously. Though smaller in spectral and temporal extent, these features have a similar $T_{B}$, implying a coherent emission process, like plasma emission. The existence of these emission features provides evidence for impulsive non-thermal energy deposition into the corona at levels lower than previously known. Evaluating their contribution to the coronal heating budget will require building up a better understanding of the frequency of occurrence and energetics of such features. Existing radio observations have been shown to be a more sensitive probe for such phenomena than X-ray observations (e.g., Ramesh et al. 2010), and these observations extend the radio sensitivity advantage by another order of magnitude.

As seen in Figure 4, much of the "narrowband" activity seen in Figure 2 maps to the location of the region 11057. Even the low-level fluctuations in the spectrum when the Sun is in a relatively quiescent state (e.g., Figure 3, bottom panel; Figure 5) manifest themselves in the image plane as flickering intensity at the location of this active region. The occurrence of many weak $\mathrm{X}$-ray flares from region 11057 around our observing period suggests a persistent low-level activity during our observations. This raises the possibility that the "narrowband" features are the radio signatures of the same magnetic field rearrangement activity: numerous small reconnection events too faint to be picked up by X-ray monitoring platforms like GOES, but with weak type-III-like radio emission strong enough to be seen by the 32T. This hypothesis is strengthened by the observation that the "narrowband" features were absent on 2010 March 29 (not shown here), a day when no X-ray activity was reported. There have been attempts to investigate the possibility of using type III solar bursts as predictors for large solar flares in the past (Jackson \& Sheridan 1979; Kane 1981). The ability to characterize type-III-like activity in much greater detail and with much higher sensitivity, demonstrated here, enhances the prospects for investigating such a connection.

Compared to the $32 \mathrm{~T}$, the MWA will provide an order of magnitude improvement in angular resolution, 16 times as much collecting area and $16^{2}$ times as many baselines. With its unprecedented high-fidelity and dynamic-range polarimetric spectroscopic imaging capability for every spectral and temporal slice, and a radio-quiet site providing seamless access to the radio frequency spectrum, the MWA will be an excellent match to the challenge of solar imaging. Every significant advance in our capability to observe the Sun has revealed that the Sun and corona are more dynamic and feature-rich than had been thought previously. Hinode and the Solar Dynamics Observatory represent the latest in that progression. The $32 \mathrm{~T}$ array is already demonstrating that we are beginning to see features at a level of detail not seen before, and we can justifiably expect the MWA to similarly add to our understanding of the solar corona and the heliosphere.
This work uses data obtained from the Murchison Radioastronomy Observatory (MRO), jointly funded by the Commonwealth Government of Australia and Western Australian State government. The MRO is managed by the CSIRO, who also provides operational support to the MWA. We acknowledge the Wajarri Yamatji people as the traditional owners of the Observatory site. Support came from the U.S. National Science Foundation (grants AST-0457585 and PHY-0835713), the Australian Research Council (grants LE0775621 and LE0882938), the U.S. Air Force Office of Scientific Research (grant FA9550-0510247), the National Collaborative Infrastructure Strategy, funded by the Australian federal government via Astronomy Australia Limited, the Smithsonian Astrophysical Observatory, the MIT School of Science, the Raman Research Institute, The Australian National University, iVEC, the Initiative in Innovative Computing and NVIDIA sponsored Center for Excellence at Harvard, and the International Centre for Radio Astronomy Research, a Joint Venture of Curtin University, and The University of Western Australia funded by the Western Australian State government.

\section{REFERENCES}

Bracewell, R. N., \& Roberts, J. A. 1954, Aust. J. Phys., 7, 615

Cohanim, B. E., Hewitt, J. N., \& de Weck, O. 2004, ApJS, 154, 705

Erickson, W. C., Kundu, M. R., Mahoney, M. J., \& Gergely, T. E. 1977, Sol. Phys., 54, 57

Jackson, B. V., \& Sheridan, K. V. 1979, Proc. Astron. Soc. Aust., 3, 383

Kai, K. 1970, Sol. Phys., 11, 310

Kane, S. R. 1981, ApJ, 247, 1113

Krucker, S., Aschwanden, M. J., Bastian, T. S., \& Benz, A. O. 1995, A\&A, 302 551

Kundu, M. R., Gergely, T. E., Szabo, A., Loiacono, R., \& White, S. M. 1986, ApJ, 308,436

Lantos, P., \& Avignon, Y. 1975, A\&A, 41, 137

Lonsdale, C. J., et al. 2009, Proc. IEEE, 97, 1497

McLean, D. J., \& Sheridan, K. V. 1985, in Solar Radiophysics: Studies of Emission from the Sun at Metre Wavelengths, ed. D. J. McLean \& N. R. Labrum (Cambridge: Cambridge Univ. Press), 443

Mercier, C., \& Chambe, G. 2009, ApJ, 700, L137

Mercier, C., Subramanian, P., Kerdraon, A., Pick, M., Ananthakrishnan, S., \& Janardhan, P. 2006, A\&A, 447, 1189

Nelson, G. J., \& Melrose, D. B. 1985, in Solar Radiophysics: Studies of Emission from the Sun at Metre Wavelengths, ed. D. J. McLean \& N. R. Labrum (Cambridge: Cambridge Univ. Press), 333

Newkirk, G., Jr. 1961, ApJ, 133, 983

Ramesh, R., Kathiravan, C., Barve, I. V., Beeharry, G. K., \& Rajasekara, G. N. 2010, ApJ, 719, L41

Robinson, P. A., \& Cairns, I. H. 2000, in Radio Astronomy at Long Wavelengths, ed. R. G. Stone et al. (Washington, DC: AGU), 37

Suzuki, S., \& Dulk, G. A. 1985, in Solar Radiophysics: Studies of Emission from the Sun at Metre Wavelengths, ed. D. J. McLean \& N. R. Labrum (Cambridge: Cambridge Univ. Press), 289

Vilmer, N., Krucker, S., \& Lin, R. P. The Rhessi Team 2002, Sol. Phys., 210, 261 